09

\title{
Распространение электромагнитных волн в электропроводящих графеноподобных углеродных наноаллотропах
}

\author{
(C) P.А. Браже, Р.М. Мефтахутдинов
}

Ульяновский государственный технический университет, 432027 Ульяновск, Россия

e-mail: mrm@ulstu.ru

(Поступило в Редакцию 10 мая 2016 г. В окончательной редакции 26 октября 2016 г.)

Исследовано распространение электромагнитных волн в углеродных графеноподобных электропроводящих кристаллах с симметрией $(\mathrm{C})_{4 v}$ и $(\mathrm{C})_{6 v}$. Показано, что в этих кристаллах могут существовать только волны ТМ поляризации. Проведен сравнительный анализ их свойств в различных структурах. Показано, что рассмотренные структуры имеют преимущество перед классическим графеном с точки зрения возбуждения в них электромагнитных волн.

DOI: 10.21883/JTF.2017.05.44452.1879

\section{Введение}

В последние годы большой интерес исследователей вызывают взаимодействия графена и графеноподобных материалов с электромагнитными волнами [1-3], причем они интересны не только с фундаментальной точки зрения, но и в связи с различными приложениями в наноэлектронике, фотонике, оптоэлектронике и др.

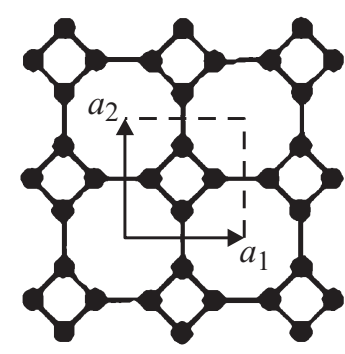

(C) 44

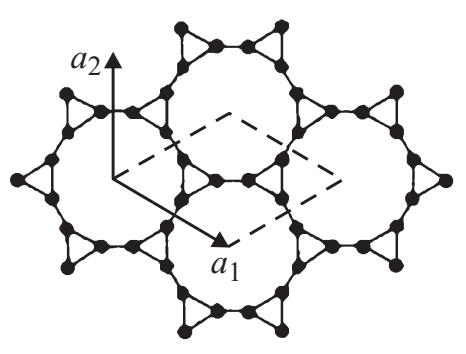

(C) $63(12)$

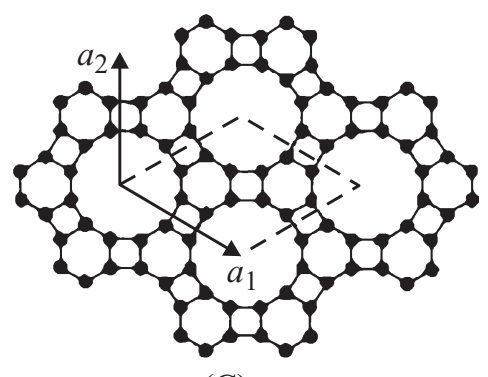

(C) 664

Рис. 1. Исследуемые структуры.
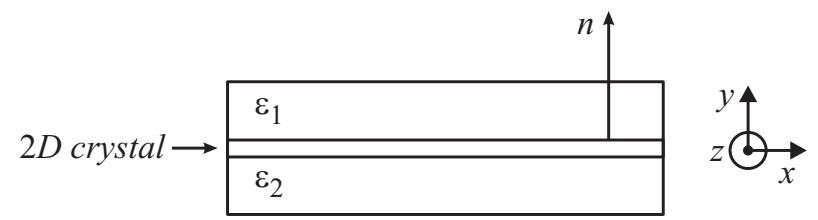

Рис. 2. Исследуемая система.
В настоящей работе исследуется распространение электромагнитных волн в углеродных графеноподобных наноаллотропах с симметрией $(\mathrm{C})_{4 v}$ и $(\mathrm{C})_{6 v}$ (рис. 1). Ранее нами были изучены их упругие и акустические [4-6], электронные и оптические свойства [7]. Рассматриваются лишь $s p^{2}$-наноаллотрпы, в которых имеются свободные электроны. Следует отметить, что существование указанных структур при комнатной температуре и их термодинамическая устойчивость пока предсказаны теоретически, однако физические свойства и возможные практические применения этих альтернативных классическому графену двумерных наноаллотропов углерода в последнее время интенсивно изучаются [8-13]. В частности, большое внимание уделяется так называемому октаграфену или Т-графену, обозначенному здесь как (C) $44[9,10,12]$.

\section{Исследуемые структуры}

На рис. 1 показаны три типа исследуемых $2 D$ супракристаллов, обладающих $s p^{2}$-гибридизацией: (C) 44 , содержащая 4 атома в элементарной ячейке (показана штриховой линией), (C) $63(12)$, содержащая 6 атомов в элементарной ячейке, и $(\mathrm{C})_{664}$ с 12 атомами. Векторы $\mathbf{a}_{1}$ и $\mathbf{a}_{2}$ - базисные. Как показывают расчеты зонной структуры [14], они принадлежат к полуметаллам.

Будем рассматривать двумерные кристаллические листы, помещенные между двумя диэлектриками с проницаемостями $\varepsilon_{1}$ и $\varepsilon_{2}$ (рис. 2).

Будем рассматривать двумерные кристаллические листы, помещенные между двумя диэлектриками с проницаемостями $\varepsilon_{1}$ и $\varepsilon_{2}$ (рис. 2).

\section{Постановка задачи}

Спектр электромагнитных волн, распространяющихся вдоль кристаллических листов, находится из решения 

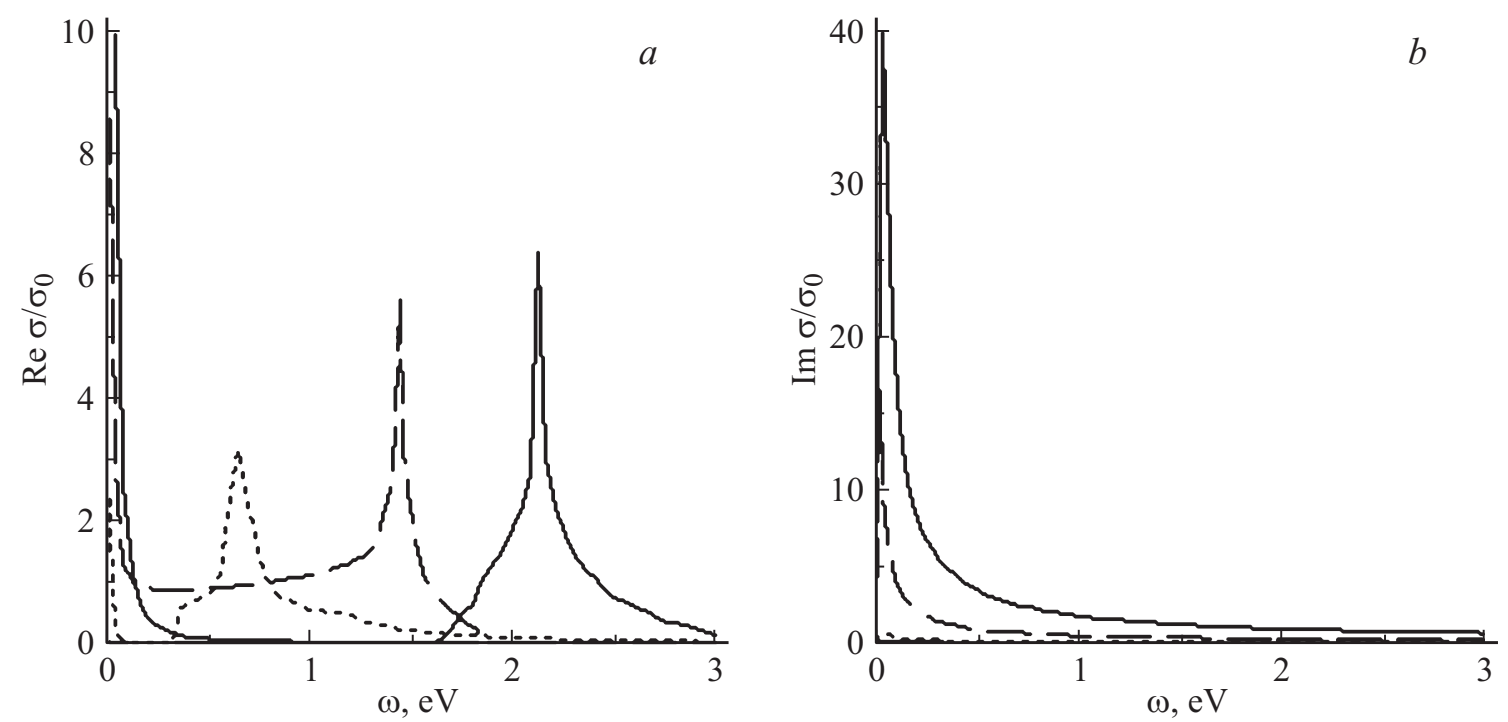

Рис. 3. Проводимость исследуемых структур: $\mathrm{C}_{44}-$ сплошная линия, $\mathrm{C}_{63(12)}-$ штриховая линия, $\mathrm{C}_{664}-$ точки. $a-$ действительная часть, $b-$ мнимая часть, $\sigma_{0}=e^{2} / 4 \hbar$.

уравнений Максвелла совместно с граничными условиями, которые имеют вид

$$
\begin{gathered}
\mathbf{E}_{1 t}=\mathbf{E}_{2 t}, \quad \mathbf{H}_{1 t}-\mathbf{H}_{2 t}=\mathbf{j}_{s} \times \mathbf{n}, \\
D_{1 n}-D_{2 n}=\rho_{s}, \quad B_{1 n}=B_{2 n},
\end{gathered}
$$

где $\mathbf{D}=\varepsilon \varepsilon_{0} \mathbf{E}, \mathbf{B}=\mu_{0} \mathbf{H}$. Плотности заряда и тока соответственно равны

$$
\rho=\rho_{s} \delta(y), \quad \mathbf{j}=\mathbf{j}_{s} \delta(y) .
$$

Здесь $\rho_{s}-$ поверхностная плотность заряда, т.е. заряд, приходящийся на единицу площади, $\mathbf{j}_{s}=\sigma \mathbf{E}_{t}-$ поверхностная плотность тока, т.е. ток на единицу длины поперечного сечения $2 D$ кристалла, дельта-функция $\delta(y)$ имеет размерность длины.

Поверхностная проводимость кристаллических листов $\sigma$ складывается из межзонной [7]

$$
\begin{aligned}
\sigma_{\text {inter }}= & \frac{e^{2}}{2 \pi m^{2} \omega} \sum_{\nu, c} \iint\left(f\left(E_{v}\right)-f\left(E_{c}\right)\right)\left|P_{\nu, c}(\mathbf{k})\right|^{2} \\
& \times \delta\left(E_{c}-E_{v}-\hbar \omega\right) d^{2} k
\end{aligned}
$$

и внутризонной [7]

$$
\sigma_{\text {intra }}=\frac{i \varepsilon_{0} \omega_{p}^{2}}{\omega+i \Gamma}
$$

проводимостей, где $\omega-$ частота падающей электромагнитной волны, $f(E)$ - функция распределения Ферми-Дирака, индексы $v$ и $c$ относятся к валентной зоне и зоне проводимости соответственно, $P_{v, c}(\mathbf{k})-$ матричные элементы оператора импульса, $\omega_{p}$ - плазменная частота, Г - коэффициент затухания.

Отметим, что квадрат плазменной частоты в (4) имеет размерность $\mathrm{m} / \mathrm{s}^{2}$ [15]. Это связано с тем, что для двумерных структур она определяется поверхностной плотностью зарядов, а не объемной, как для трехмерных структур.

Расчет частотной зависимости действительной и мнимой частей полной проводимости исследуемых структур проводился при следующих значениях параметров: $E_{f}=0 \mathrm{eV}, \hbar \Gamma=10 \mathrm{meV}$ и $k T=25 \mathrm{meV}(T=300 \mathrm{~K})$.

Что касается $\Gamma$, то теоретических моделей расчета коэффициента затухания пока не существует [15], но в силу важности этого параметра для внутризонной проводимости мы принимаем его равным $10 \mathrm{meV}$. Это связано с тем, что в графенах основным механизмом рассеяния носителей заряда является электрон-фононное взаимодействие, и время релаксации можно с достаточной точностью считать равным $\tau=0.5 \cdot 10^{-13} \mathrm{~s}[1]$, что, примерно, соответствует $10 \mathrm{meV}$.

Результаты расчета проводимости представлены на рис. 3.

\section{ТМ волны}

Дисперсионное соотношение, описывающее поверхностные ТМ волны, известные также как плазмонполяритоны, имеет вид

$$
\frac{\varepsilon_{1}}{q_{1}}+\frac{\varepsilon_{2}}{q_{2}}=-i \frac{\sigma}{\varepsilon_{0} \omega}
$$

где коэффициенты $q_{1}$ и $q_{2}$ равны

$$
q_{1}=\sqrt{k^{2}-\omega^{2} \varepsilon_{1} / c^{2}}, \quad q_{2}=\sqrt{k^{2}-\omega^{2} \varepsilon_{2} / c^{2}} .
$$

Для диапазона частот от 0 до $15 \mathrm{meV}$ уравнение (5) решалось численно с использованием пакета Maple 17. На рис. 4 показаны результаты расчетов для всех 

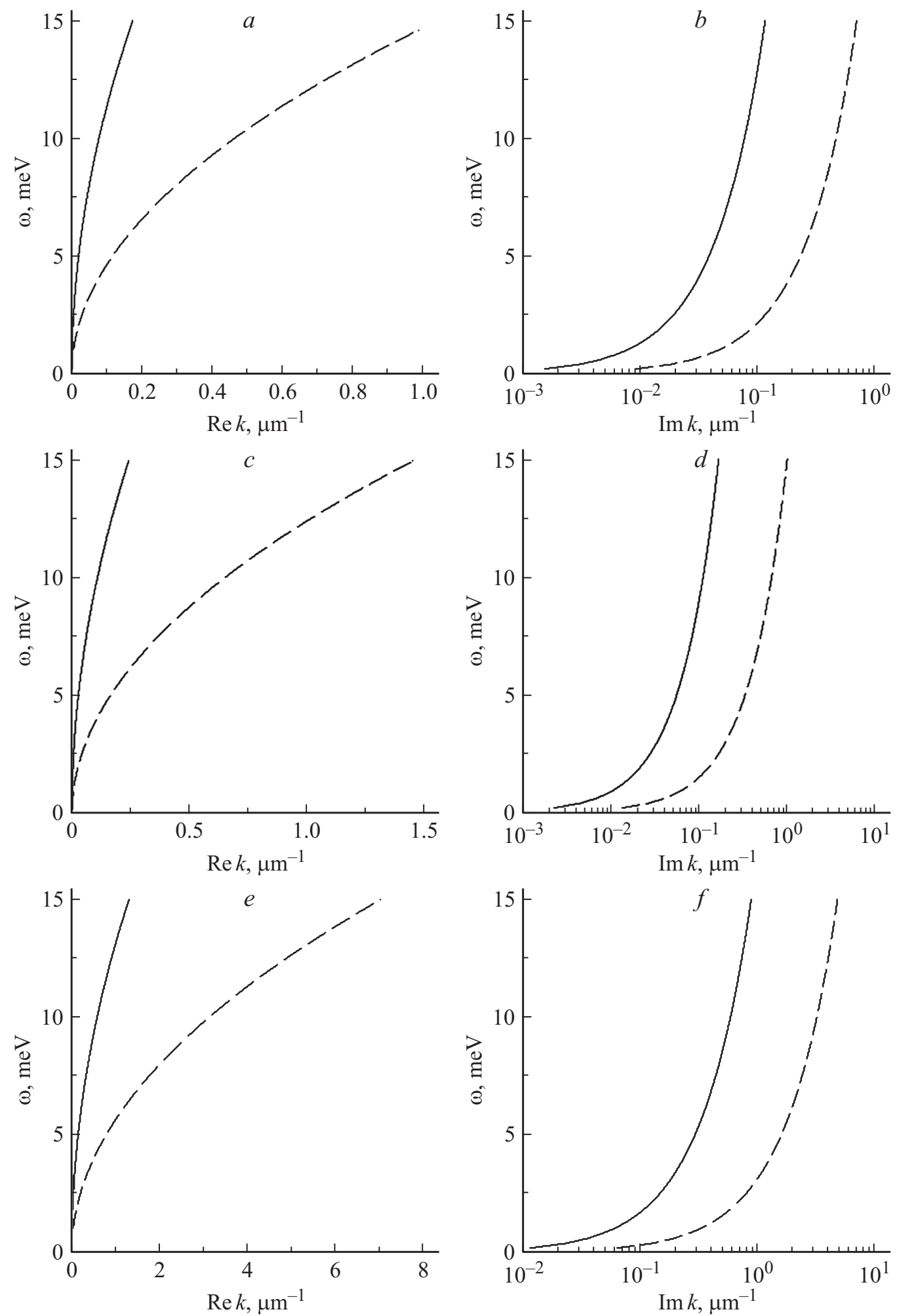

Рис. 4. Дисперсионные зависимости для волн ТМ поляризации: $a, b-$ для $\mathrm{C}_{44}, c, d-$ для $\mathrm{C}_{63(12)}, e, f-$ для $\mathrm{C}_{664}$. Сплошная линия для $\varepsilon_{1}=\varepsilon_{2}=1$, штриховая для $\varepsilon_{1}=1, \varepsilon_{2}=11$. Мнимые части волнового числа отображены в логарифмическом масштабе.

структур. Сплошная линия соответствует „подвешенным“ структурам, т. е. $\varepsilon_{1}=\varepsilon_{2}=1$, штриховая $-\varepsilon_{1}=1$, $\varepsilon_{2}=11$ (кремний в качестве подложки). Из рисунка видно, что поверхностные электромагнитные волны суще- ствуют во всем представленном диапазоне частот. Для сравнения волн в разных структурах для частоты $4 \mathrm{meV}$, что соответствует $1 \mathrm{THz}$, были рассчитаны следующие параметры: фазовая скорость $\omega / k$, длина распростране- 
Параметры волн ТМ поляризации в различных структурах

\begin{tabular}{c|c|c|c|c}
\hline Структура & $\varepsilon$ & $v_{p h}, \times 10^{5}, \mathrm{~m} / \mathrm{s}$ & $1 / \operatorname{Im}(k), \mu \mathrm{m}$ & $1 / \operatorname{Re}(q), \mu \mathrm{m}$ \\
\hline \multirow{2}{*}{$\mathrm{C}_{44}$} & 1 & 3.25 & 32.26 & 95.28 \\
\cline { 2 - 5 } & 11 & 0.54 & 5.41 & 14.25 \\
\hline \multirow{2}{*}{$\mathrm{C}_{664}$} & 1 & 0.43 & 4.35 & 10.90 \\
\cline { 2 - 5 } & 11 & 0.08 & 0.80 & 2.01 \\
\hline \multirow{2}{*}{$\mathrm{C}_{63(12)}$} & 1 & 2.35 & 23.25 & 64.20 \\
\cline { 2 - 5 } & 11 & 0.39 & 3.87 & 9.98 \\
\hline \multirow{2}{*}{ Графен } & 1 & 380.00 & 2.50 & 6.24 \\
\cline { 2 - 5 } & 11 & 63.30 & 0.42 & 1.04
\end{tabular}

ния $1 / \operatorname{Im} k$, расстояние в поперечном направлении, на котором поле волны уменьшается в $e$ раз $-1 / \operatorname{Re}\left(q_{1}\right)$ для среды с диэлектрической проницаемостью $\varepsilon_{1}$, и $1 / \operatorname{Re}\left(q_{2}\right)$ с диэлектрической проницаемостью $\varepsilon_{2}$. Эти же самые параметры были рассчитаны для поверхностных плазмон-поляритонов в чистом графене $\left(E_{f}=0 \mathrm{eV}\right)$. При этом его проводимость определялась моделью Друде, в рассматриваемом диапазоне частот такое упрощение вполне оправдано.

Полученные результаты приведены в таблице. Из нее видно, что поверхностные электромагнитные волны в (C) 44 имеют бо́льшую скорость распространения, бо́льшую глубину проникновения, но меньшую локализацию, чем в других исследуемых структурах. Если сравнивать с классическим графеном, то фазовая скорость поверхностных плазмонов и их локализация в исследуемых структурах существенно меньше, чем в графене, однако глубина проникновения значительно больше. Последнее может иметь существенное преимущество с точки зрения их использования в устройствах обработки и передачи информации.

\section{ТЕ волны}

Решение уравнений Максвелла с граничными условиями дает следующее дисперсионное уравнение для ТЕ волн

$$
\sqrt{k^{2}-\varepsilon_{1} \frac{\omega^{2}}{c^{2}}}-\sqrt{k^{2}-\varepsilon_{2} \frac{\omega^{2}}{c^{2}}}=i \omega \mu_{0} \sigma .
$$

Полученное уравнение допускает действительные решения только в том случае, если мнимая часть проводимости отрицательна. Из рис. 3 видно, что вплоть до $3 \mathrm{eV}$ для всех исследуемых структур мнимая часть проводимости положительна, и, следовательно, ТЕ моды в них распространяться не могут.

\section{Заключение}

В настоящей работе было рассмотрено распространение электромагнитных волн в графеноподобных проводящих структурах, расположенных как на кремниевой подложке, так и в подвешенном состоянии. Показано, что в исследуемых кристаллах могут существовать плазмонные моды только ТМ поляризации. Анализ исследованных электромагнитных волн в указанных кристаллах показал, что поверхностные электромагнитные волны в (C) 44 (рис. 1) имеют бо́льшую скорость распространения, бо́льшую глубину проникновения, но меньшую локализацию по сравнению с другими исследуемыми структурами. Исследуемые структуры имеют некоторое преимущество с точки зрения возбуждения в них электромагнитных волн перед графеном, поскольку глубина проникновения волн в них существенно больше.

Работа выполнена в рамках государственного задания Министерства образования и науки РФ 2014/232 (проект № 1742).

\section{Список литературы}

[1] Kotov O.V., Kol' chenko M.A., Lozovik Yu.E. // Opt. Express. 2013. Vol. 21. N 11. P. 13533-13546.

[2] Буслаев П.И., Иорш И.В., Шадривов И.В. и др. // Письма в ЖЭТФ. 2013. Т. 97. Вып. 9. С. 619-623.

[3] Колесников А.А., Лозовик Ю.Е. // Труды МФТИ. 2013. Т. 5. N 1. C. 53-59.

[4] Браже Р.А., Каренин А.А., Кочаев А.И., Мефтахутдинов Р.М. // ФТТ. 2011. Т. 53. Вып. 7. С. 1406-1408.

[5] Браже Р.А., Кочаев А.И., Мефтахутдинов Р.М. // ФТТ. 2011. Т. 53. Вып. 8. С. 1614-1617.

[6] Браже Р.А., Кочаев А.И. // ФТТ. 2012. Т. 54. Вып. 8. C. $1512-1514$.

[7] Браже Р.А., Мебтахутдинов Р.М. // ЖТФ. 2016. Т. 86. Вып. 5. C. 112-117.

[8] Enyashin A.N., Ivanovskii A.L. // Phys. Stat. Sol. B. 2011. Vol. 248. N 8. P. 1879-1883.

[9] Sheng X.-L. et al. // J. Appl. Phys. 2012. Vol. 112. P. 074315.

[10] Liu Y. et al. // Phys. Rev. Lett. 2012. Vol. 108. P. 225505.

[11] Беленков Е.А., Грешняков В.А. // ФТТ. 2013. Т. 55. Вып. 8. C. $1640-1650$.

[12] Подливаев А.И., Опенов Л.А. // ФТТ. 2013. Т. 55. Вып. 12. C. 2464-2467.

[13] Fthenakis Z.G., Lathiotakis N.N. // Phys. Chem. Chem. Phys. 2015. Vol. 17. P. $16418-16427$.

[14] Бражее Р.А., Мефбтахутдинов Р.М., Фатхутдинова К.X. // Изв. вузов. Поволжский регион. 2015. № 1. C. $130-139$.

[15] Pedersen T.G. Electric, optical and magnetic properties of nanostructures. Aalborg university, 2015. 339 p. 